\title{
Guanosine exerts neuroprotective effects by reversing mitochondrial dysfunction in a cellular model of Parkinson's disease
}

\author{
DA-WEI LI ${ }^{1}$, MIN YAO $^{2}$, YAN-HUA DONG ${ }^{2}$, MIN-NA TANG $^{2}$, \\ WEI CHEN ${ }^{2}$, GUANG-REN LI ${ }^{2}$ and BI-QUAN SUN ${ }^{2}$ \\ ${ }^{1}$ Department of Neurology, Affiliated Hospital of Beihua University, Jilin City, Jilin; \\ ${ }^{2}$ Department of Neurology, The Third Hospital of Jilin University, Changchun, Jilin, P.R. China
}

Received April 19,2014; Accepted August 7, 2014

DOI: $10.3892 /$ ijmm.2014.1904

\begin{abstract}
The mitochondria are the most important cytoplasmic organelles in determining cell survival and death. Mitochondrial dysfunction leads to a wide range of disorders, including neurodegenerative diseases. The central events in the mitochondrial-dependent cell death pathway are the activation of the mitochodrial permeability transition pore (mPTP) and the disruption of mitochondrial membrane potential, which cause the release of apoptogenic molecules and finally lead to cell death. This is thought to be at least partly responsible for the loss of dopaminergic neurons in Parkinson's disease (PD); thus, the attenuation of mitochondrial dysfunction may contribute to alleviating the severity and progression of this disease. Guanosine is a pleiotropic molecule affecting multiple cellular processes, including cellular growth, differentiation and survival. Its protective effects on the central nervous system and and on several cell types by inhibiting apoptosis have been shown in a number of pathological conditions. This study aimed to analyze the ability of guanosine to protect neuronal PC12 cells from the toxicity induced by 1-methyl-4-phenylpyridinium $\left(\mathrm{MPP}^{+}\right)$, the active metabolite of 1-methyl-4-phenyl-1,2,3,6tetrahydropyridine (MPTP), which mediates selective damage to dopaminergic neurons and causes irreversible Parkinson-like symptoms in humans and primates. Our results demonstrated that the apoptosis of $\mathrm{PC} 12$ cells induced by $\mathrm{MPP}^{+}$was significantly prevented by pre-treatment for $3 \mathrm{~h}$ with guanosine. In addition, guanosine attenuated the $\mathrm{MPP}^{+}$-induced collapse of mitochondrial transmembrane potential and prevented the
\end{abstract}

Correspondence to: Professor Guang-Ren Li, Department of Neurology, The Third Hospital of Jilin University, 126 Xiantai Street, Changchun, Jilin 130033, P.R. China

E-mail: Lgren18@hotmail.com

Abbreviations: PD, Parkinson's disease; MPTP, 1-methyl-4-phenyl1,2,3,6-tetrahydropyridine; $\mathrm{MPP}^{+}$, 1-methyl-4-phenylpyridinium; mPTP, mitochodrial permeability transition pore; DCF, 2'7'-dichlorodihydrofluorescein; Bcl-2, B-cell lymphoma 2; ROS, reactive oxygen species

Key words: Parkinson's disease, mitochondrial dysfunction, apoptosis, guanosine, neuroprotection, 1-methyl-4-phenylpyridinium sebsequent activation of caspase-3, thereby protecting dopaminergic neurons against mitochondrial stress-induced damage.

\section{Introduction}

Parkinson's disease (PD) is a common neurodegenerative disorder characterized by the gradually progressive and selective loss of dopaminergic neurons in the substantia nigra (1). The progressive loss of dopaminergic neurons is a complex process, and multiple pathological events are involved in this process (2-6). While the underlying mechanisms of nigrostriatal dopaminergic neuron degeneration are not yet completely understood, accumulating evidence indicates that mitochondrial dysfunction may be a central event in neurodegenerative diseases (7-9). The mitochondria are multifunctional organelles that are important for living cells. Mitochondrial dysfuntion has a multitude of consequences for cells, including apoptosis (10). The activation of the mitochondrial permeability transition pore (mPTP) and the collapse of the mitochondrial membrane potential may be major contributors to mitochondrial-dependent cell death and at least partly reponsible for the pathogenesis of PD and several other neurodegenerative disorders (7-9).

PC12 cells treated with 1-methyl-4-phenylpyridinium $\left(\mathrm{MPP}^{+}\right)$provide a reliable in vitro model for investigating the pathogenesis of $\mathrm{PD}$. $\mathrm{MPP}^{+}$is an active metobolite of the neurotoxin, 1-methyl-4-phenyl-1,2,3,6-tetrahydropyridine (MPTP), which is known to selectively kill dopaminergic neurons and cause irreversible Parkinson-like symptoms in humans and primates (11-13). MPTP is a lipophilic molecule that can rapidly cross the blood-brain barrier; it is subsequently oxidized in the brain to its toxic metabolite, $\mathrm{MPP}^{+}$, by type $\mathrm{B}$ monoamine oxidase (14). The neurotoxic action of $\mathrm{MPP}^{+}$is related to the activation of the MPTP and the collapse of mitochondrial membrane potential through oxidative damage, which together initiate the downstream apoptotic pathway, including the release of cytochome $\mathrm{c}$ and the activation of caspases, finally leading to neuronal cell death (7-9). Damage to the mitochondria is considered as an initial and irreversible step towards apoptosis; thus, mitochondrial-targeted therapeutic strategies may be a promising treatment for PD. Guanosine, a non-adenine-based purine, is an intercellular signaling molecule affecting multiple cellular processes, including cellular growth, differentiation and survival (15-17). In multiple cell types, it exerts protective 
effects against apoptosis induced by a number of agents, such as staurosporine (18), $\beta$-amyloid (19) and MPTP (20). The neuroprotective effects of guanosine in the central nervous system have also been recognized (15). The present study was designed to investigate the effects of guanosine on $\mathrm{MPP}^{+}$-induced apoptosis in PC12 cells and the underlying mechanisms for these actions. Our results demonstrated that guanosine effectively prevented $\mathrm{MPP}^{+}$-induced $\mathrm{PC} 12$ cell apoptosis by stabilizing the mitochondrial membrane potential and attenuating the subsequent activation of caspases. In addition, guanosine inhibited the production of reactive oxygen species (ROS) and increased the expression levels of glutathione (GSH), further supporting the protective role of guanosine in oxidative conditions. Overall, these findings indicate the protective role of guanosine in mitochondrial stress-induced dopaminergic neuronal damage, thus providing potential effective strategies for the treatment of PD.

\section{Materials and methods}

Drugs and chemicals. All reagents and chemicals were purchased from Sigma-Aldrich (St. Louis, MO, USA) unless stated otherwise.

PC12 cell cultures. The PC12 cells were obtained from the Cell Bank of the Chinese Academy of Sciences (Shanghai, China) and maintained in high glucose Dulbecco's modified Eagle's medium (DMEM) supplemented with $10 \%$ heat-inactivated fetal bovine serum, $4.00 \mathrm{mM}$ L-glutamine, $100 \mathrm{U} / \mathrm{ml}$ of penicillin and $100 \mu \mathrm{g} / \mathrm{ml}$ of streptomycin (Gibco, Grand Island, NY, USA). The cultures were maintained in a humidified $5 \% \mathrm{CO}_{2}$ atmosphere at $37^{\circ} \mathrm{C}$. The culture medium was changed every 3-4 days and the cells were seeded at a density of 30,000 cells $/ \mathrm{cm}^{2}$.

Cell viability assay. Cell viability was measured using the modified 3-(4,5-dimethylthiazol-2-yl)-2,5-diphenyltetrazolium bromide (MTT), a mitochondrial dye which is converted into a blue formazan product by mitochondrial dehydrogenases in metabolically active cells. The PC12 cells were plated at a density of 30,000 cells $/ \mathrm{cm}^{2}$ in 96 -well plates and incubated for $24 \mathrm{~h}$. To assess the neuroprotective effects of guanosine on $\mathrm{MPP}^{+}$-induced toxicity in PC12 cells, the cells were pre-treated with various concentrations of guanosine $(0.01-1,000 \mu \mathrm{M})$ for $3 \mathrm{~h}$ and were then exposed to $500 \mu \mathrm{M} \mathrm{MPP}^{+}$for $72 \mathrm{~h}$, under optimal conditions for the assessment of the neuroprotective effects as previously described (21). MTT (5 mg/ml) solution was added to the wells and the cells were incubated for $4 \mathrm{~h}$. Subsequently, the culture medium was removed, and dimethyl sulfoxide was added to each well to solubilize the formazan into a colored solution. The absorbance of colored solution was measured at $570 \mathrm{~nm}$ using a microplate reader (Epoch; BioTek, Winooski, VT, USA). The results were expressed as the percentage of the absorbance of the control culture wells. Based on these results, we used guanosine at a dose of $10 \mu \mathrm{M}$ in all the subsequent experiments.

Nuclear staining assay. The morphological signs of apoptosis induced by $\mathrm{MPP}^{+}$were detected using acridine orange (AO)/ethidium bromide (EB) staining of the PC12 cells. The cells were plated in 6-well plates at a density of 30,000 cells $/ \mathrm{cm}^{2}$ and were incubated in DMEM medium at $37^{\circ} \mathrm{C}$. After $3 \mathrm{~h}$ of pre- treatment with guanosine $(10 \mu \mathrm{M}), \mathrm{MPP}^{+}(500 \mu \mathrm{M})$ was added to the medium for $72 \mathrm{~h}$. The cells were washed and resuspended in phosphate-buffered saline (PBS) and AO/EB was added at a final concentration of $1 \mu \mathrm{g} / \mathrm{ml}$. Subsequently, the number of apoptotic cells was randomly counted under a fluorescence microscope (IX71; Olympus, Tokyo, Japan). Viable cells with intact structures stained with AO only showed bright green nuclear staining; the early apoptotic cells were bright green and later apoptotic cells were red-orange with condensed chromatin. The number of apoptotic cells is expressed as a percentage of the total cells counted.

Measurement of apoptosis in cells. Apoptosis was assessed by measuring DNA fragmentation with single-stranded DNA (ssDNA) apoptosis enzyme-linked immunosorbent assay (ELISA) kits (Chemicon International, Temecula, CA, USA) according to the manufacture's instructions. The cells plated at a concentration of 30,000 cells $/ \mathrm{cm}^{2}$ were cultured for $24 \mathrm{~h}$, followed by treatment with $10 \mu \mathrm{M}$ guanosine prior to the addition of $500 \mu \mathrm{M} \mathrm{MPP}^{+}$for $3 \mathrm{~h}$. The cells were washed 3 times with PBS and formamide was then added which selectively denaturates DNA in apoptotic cells. Anti-ssDNA monoclonal antibody and peroxidase-conjugated secondary antibody were then added to the cells; the ssDNA was then measured at $450 \mathrm{~nm}$ using a microplate reader (Epoch; BioTek).

Measurement of mitochondrial transmembrane potential. Mitochondrial membrane potential is a key indicator of mitochondrial function and cell death or injury, which can be detected using the mitochondrial dye, 3,3-dihexyloxacarbocyanine iodide $\left[\mathrm{DiOC}_{6}(3)\right]$. This dye is a lipophilic fluorescent stain and becomes highly fluorescent when incorporated into membranes. The cells at a concentration of 30,000 cells $/ \mathrm{cm}^{2}$ were cultured in 24-well plates for $24 \mathrm{~h}$, followed by treatment with $10 \mu \mathrm{M}$ guanosine prior to the addition of $500 \mu \mathrm{M} \mathrm{MPP}^{+}$for $3 \mathrm{~h}$. Following $72 \mathrm{~h}$ of incubation, $1 \mathrm{ml}$ of serum-free culture medium containing $\mathrm{DiOC}_{6}(3)$ was added to each well with the final concentration of $1 \mu \mathrm{M}$, and the cells were cultured in a humidified incubator for $15 \mathrm{~min}$. The cells were collected and centrifuged at $1,000 \mathrm{x} \mathrm{g}$ for $5 \mathrm{~min}$, and the cell pellets were resuspended in PBS containing $0.5 \mathrm{mM}$ EDTA. The intensity of $\mathrm{DiOC}_{6}(3)$ fluorescence was recorded using a flow cytometer (Becton-Dickinson, San Diego, CA, USA).

Western blot analysis. Following treatment, the PC12 cells were collected and lysed with cell lysis solution containing $4 \%$ sodium dodecyl sulfate (SDS), $2 \mathrm{mM}$ EDTA and $50 \mathrm{mM}$ Tris-HCl, $\mathrm{pH}$ 6.8. Equal amounts of protein were loaded onto a $12 \%$ SDS-polyacrylamide gel. Following electrophoretic separation, the polyacrylamide gels were transferred onto PVDF transfer membranes (Amersham Biosciences, Uppsala, Sweden). The membranes were incubated in Tris-buffered saline/Tween-20 (TBST) supplemented with 5\% fat-free milk for $1 \mathrm{~h}$ to block non-specific binding. The blots were incubated using rabbit anti-Bax, anti-B-cell lymphoma 2 (Bcl-2) antibodies. Horseradish peroxidase (HRP)-conjugated anti-rabbit antibodies were used as the secondary antidodies.

Measurement of ROS production. Intracellular ROS produced during the inhibition of mitochondrial complex I was detected 


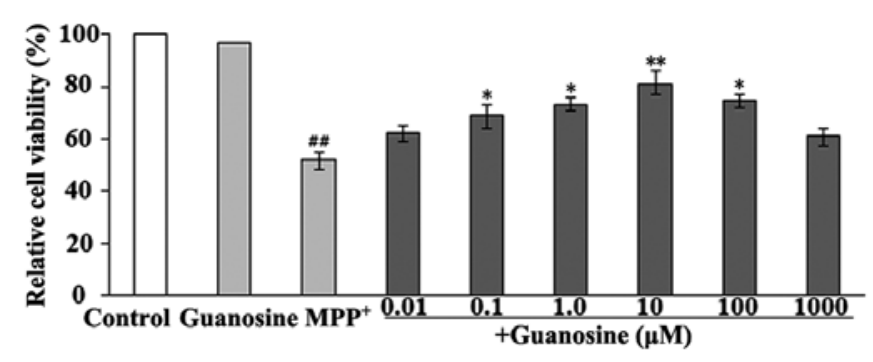

Figure 1. Effect of guanosine on 1-methyl-4-phenylpyridinium $\left(\mathrm{MPP}^{+}\right)$ induced toxicity in PC12 cells. PC12 cells were pre-treated with guanosine $(0.01-1,000 \mu \mathrm{M})$ for $3 \mathrm{~h}$, and then exposed to $500 \mu \mathrm{MMPP}^{+}$for $72 \mathrm{~h}$. Cell viability was determined by MTT assay as described in the Materials and methods. Data are presented as the means \pm standard error of the mean (SEM) of 3 independent experiments performed in sixplicate. ${ }^{\# \#} \mathrm{p}<0.01 \mathrm{vs}$. control and ${ }^{*} \mathrm{p}<0.05$ or ${ }^{* *} \mathrm{p}<0.01$ compared to treatmen with $\mathrm{MPP}^{+}$.

using 2'-7'-dichlorofluorescein diacetate (DCFH-DA). This is a non-fluorescent cell-permeating compound that can easily diffuse into cells and be converted into dichlorofluorescin (DCFH) by intracellular esterase. DCFH is then trapped within the cell and oxidized into fluorescent dichlorofluorescein (DCF) by intracellular ROS. Following treatment, the cells were incubated in BSA-free DMEM with DCFH-DA at a final concentration of $20 \mu \mathrm{M}$ for $30 \mathrm{~min}$ at $37^{\circ} \mathrm{C}$. Thereafter, 10,000 cells of each group were analyzed by flow cytometry using the FL1 flow cytometer detection channels. The excitation wavelength was $485 \mathrm{~nm}$ and the reading was performed at $530 \mathrm{~nm}$.

Measurement of GSH levels. GSH levels were measured using GSH reductase, as previously described (22). Briefly, following centrifugation and washing with PBS, the cells were dissovled with 2\% 5-sulfosalicylic acid and incubated in $100 \mu \mathrm{l}$ of the reaction mixture containing $20 \mathrm{mM}$ sodium EDTA, $600 \mu \mathrm{M}$ nicotinamide adenine dinucleotide phosphate (NADPH), $12 \mathrm{mM} \mathrm{5,5'-dithiobis(2-nitrobenzoic} \mathrm{acid)} \mathrm{and}$ $105 \mathrm{mM} \mathrm{NaH}_{2} \mathrm{PO}_{4}$. GSH reductase was added to each well, and the cells were cultured in a humidified incubator for $10 \mathrm{~min}$. Absorbance was measured at $450 \mathrm{nM}$, and the calibration curve was performed with standard GSH solutions. The results are expressed as percentages of the control condition.

Evaluation of caspase-3 activity. Caspase-3 activity was measured using an ApoAlert caspase-3 assay kit according to the manufacturer's instructions. Briefly, the cells were lysed and centrifuged at $1,000 \mathrm{x} \mathrm{g}$ for $10 \mathrm{~min}$, then the supernatant was added to the reaction mixture containing dithiothreitol and caspase-3 substrate (N-acetyl-Asp-Glu-Val-Asp p-nitroanilide). The cells were incubated for $1 \mathrm{~h}$ at $37^{\circ} \mathrm{C}$, and the absorbance of the chromophore $\mathrm{p}$-nitroanilide produced was measured at $450 \mathrm{~nm}$. The standard curves were obtained from the absorbance of p-nitroanilide standard reagent diluted with cell lysis buffer. One unit of the enzyme was defined as the activity producing $1 \mathrm{nmol}$ of $\mathrm{p}$-nitroanilide.

Statistical analysis. Data are expressed as the means \pm standard error of the mean (SEM). Statistical analysis was performed by one-way analysis of variance, followed by Dunnett's multiple-comparisons test. Differences between mean values were considered statistically different at $\mathrm{p}<0.05$.

\section{Results}

Guanosine reduces the $M P P^{+}$-induced loss of cell viability. The ability of guanosine to reverse the cytotoxicity to PC12 cells induced by $\mathrm{MPP}^{+}$was investigated using MTT, which is a mitochondrial dye and can be converted into a blue formazan product by mitochondrial dehydrogenases; therefore, it can partially detect the levels of metabolically active cells. The measurements revealed a significant decrease in the viability of the PC12 cells following exposure to $500 \mu \mathrm{M} \mathrm{MPP}^{+}$for $72 \mathrm{~h}$; however, the cells treated with guanosine alone did not show a decrease in cell viability. Pre-treatment with $10 \mu \mathrm{M}$ guanosine significantly decreased the $\mathrm{MPP}^{+}$-induced cytotoxicity (Fig. 1).

Guanosine attenuates MPP ${ }^{+}$-induced apoptosis in PC12 cells. To determine whether guanosine prevents $\mathrm{MPP}^{+}$-induced apoptosis in PC12 cells, AO/EB and DNA fragmentation assays were performed. Apoptosis is a process of programmed cell death characterized by a series of distinct nuclear morphological changes. These changes can be detected by AO/EB staining. This assay identified 3 types of cells under a fluorescence microscope: live cells (green), early apoptotic cells (bright green with condensed chromation) and later apoptotic cells (red-orange with condensed chromation). The administration of guanosine alone did not induce changes in the number of apoptotic cells, while the administration of $\mathrm{MPP}^{+}$significantly increased the number of apoptotic cells compared to the control group $(\mathrm{p}<0.01)$. Pre-treatment with $10 \mu \mathrm{M}$ guanosine significantly decreased the number of apoptotic cells induced by exposure to $\mathrm{MPP}^{+}$( $\mathrm{p}<0.01$; Fig. 2), indicating that guanosine plays an antiapoptotic role. To clarify the neuroprotective role of guanosine on $\mathrm{MPP}^{+}$-induced toxicity in PC12 cells, DNA fragmentation, a marker of late apoptosis, was further investigated by ssDNA assay. The results revealed that the increase in DNA fragmentation induced by exposure to $\mathrm{MPP}^{+}$was markedly attenuated by pre-treatment with guanosine (Fig. 3), supporting the protective role of guanosine in conditions of oxidative stress.

Guanosine modulates Bax and Bcl-2 protein expression. Bax and $\mathrm{Bcl}-2$ are key members of the Bcl-2 family of proteins that contribute to the opening of MPTP, leading to the induction of apoptosis. To investigate the changes in Bax and Bcl-2 protein expression levels, western blot analysis was performed on the untreated cells and the cells treated with $500 \mu \mathrm{M} \mathrm{MPP}^{+}$ alone or $500 \mu \mathrm{M} \mathrm{MPP}^{+}$in the presence of $10 \mu \mathrm{M}$ of guanosine. The administration of $\mathrm{MPP}^{+}$significantly increased the levels of Bax expression and decreased Bcl-2 expression. These changes were be markedly reversed by pre-treatment with guanosine. Treatment with guanosine alone did not induce changes in the expression levels of these proteins (Fig. 4), thus further demonstrating the protective role of guanosine in mitochondrial-stress induced cell damage.

Guanosine prevents the $M P P^{+}$-induced collapse of mitochondrial transmembrane potential. Mitochondrial membrane potential maintenance is essential for living cells, and its collapse is a key event in the activation of the mitochondrial-dependent 

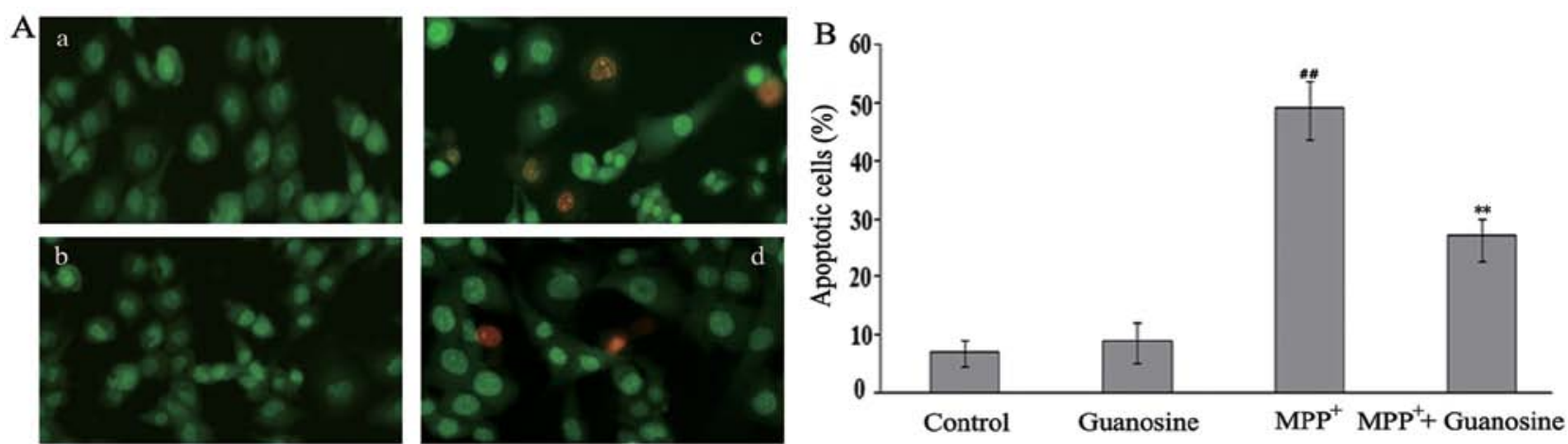

Figure 2. (A) Effects of guanosine on 1-methyl-4-phenylpyridinium (MPP+)-induced neuronal cell apoptosis were analyzed by acridine orange (AO)/ethidium bromide (EB) assay. (a) Control; (b) treatment with $10 \mu \mathrm{M}$ guanosine; (c) treatment with $500 \mu \mathrm{M} \mathrm{MPP}^{+}$; (d) treatment with $10 \mu \mathrm{M}_{\text {guanosine plus } 500 \mu \mathrm{M} \text { MPP }}^{+}$. (B) Histograms show percentages of values in total cells. Data are presented as the means \pm standard error of the mean (SEM). $n=3$. ${ }^{\# \#} \mathrm{p}<0.01 \mathrm{vs}$. control and ${ }^{* *} \mathrm{p}<0.01$ compared to treatment with $\mathrm{MPP}^{+}$.

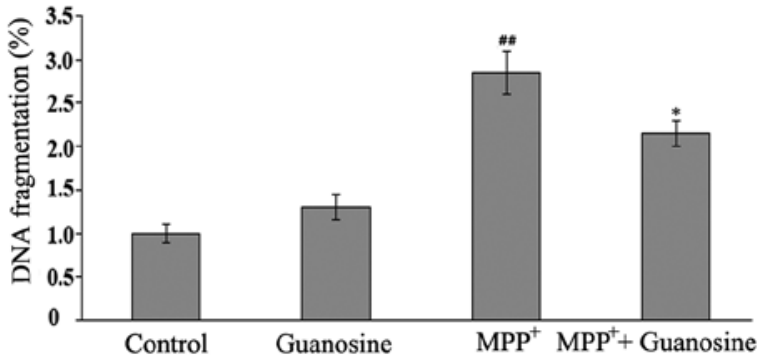

Figure 3. Effects of guanosine on 1-methyl-4-phenylpyridinium (MPP+)induced apoptosis of PC12 cells was determined by single-stranded DNA assay. The histograms show percentages of values in total cells. Data are presented as the means \pm standard error of the mean (SEM). $n=3 . " \# p<0.01$ vs. control and " $\mathrm{p}<0.01$ compared to treatmen with $\mathrm{MPP}^{+}$.

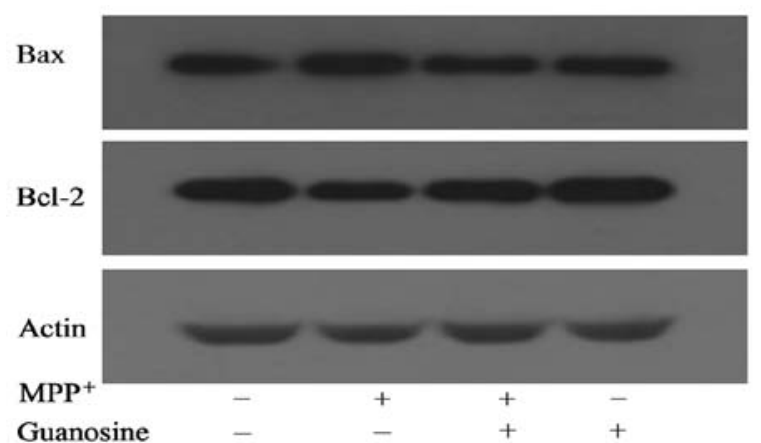

Figure 4. Effects of guanosine on the levels of Bax and Bcl-2 protein expression in 1-methyl-4-phenylpyridinium ( $\left.\mathrm{MPP}^{+}\right)$-exposed $\mathrm{PC} 12$ cells. PC12 cells were either untreated, or treated with $500 \mu \mathrm{M} \mathrm{MPP}^{+}$alone or $500 \mu \mathrm{M} \mathrm{MPP}$ in the presence of $10 \mu \mathrm{M}$ guanosine. The protein expression levels of Bax and Bcl-2 were determined by western blot analysis, and actin levels were measured as a loading control.

pathway. The collapse of mitochondrial transmembrane potential was assessed by measuring the response to the mitochondrial dye, $\mathrm{DiOC}_{6}(3)$, which is converted into a highly green fluorescent dye following incorporation into mitochondrial membranes, thereby allowing the qualitative assessment of mitochondrial membrane potential. The administration of $\mathrm{MPP}^{+}$in comparison with the control cells induced a significant decrease in fluorescence intensity, indicating the increasing percentage of the cells with collapse of mitochondrial membrane potential. The results also revealed a marked reduction in the number of cells with the collapse of mitochondrial membrane potential, when guanosine was administered prior to exposure to $\mathrm{MPP}^{+}$; no significant change was observed following treatment with guanosine alone (Fig. 5). These results suggest that the mitochondrial dysfunction induced by $\mathrm{MPP}^{+}$can be partly restored by the administration of guanosine.

Guanosine inhitis the MPP ${ }^{+}$-induced production of ROS. The levels of ROS production were evaluated by flow cytometry with DCFH-DA. DCFH-DA is a stable compound that can easily diffuse into cells, where it is converted into DCFH by intracellular esterase. DCFH is then trapped within cells and oxidized to highly fluorescent DCF by intracellular ROS; thus, the intensity of fluorescence produced by DCF may reflect an intracellular oxidative state.

The administration of guanosine alone, compared with the control group, did not elicit changes in the levels of DCFH oxidation. The administration of $\mathrm{MPP}^{+}$induced a significant increase in DCFH oxidation in the PC12 cells, which was markedly reversed by pre-treatment with guanosine (Fig. 6A), thus indicating that guanosine may play an antioxidant role.

Guanosine reverses the $\mathrm{MPP}^{+}$-induced reduction in GSH levels. GSH protein is a major non-enzymatic antioxidant that plays a crucial role in protecting neurons from oxidative damage in the central nervous system (23). To assess the protective role of guanosine in $\mathrm{MPP}^{+}$-induced oxidative damage, the levels of GSH were measured in the PC12 cells. The administration of $\mathrm{MPP}^{+}$in comparison with the control cells induced a significant decrease in GSH levels; this effect was markedly reversed by pre-treatment with guanosine. The administration of guanosine alone did not elicit any changes in the levels of GSH (Fig. 6B).

Guanosine reduces caspase-3 activity. Caspase-3 is an effector caspase that cleaves a wide range of signal transduction proteins in the apoptotic process (24). To determine whether guanosine protects neuronal $\mathrm{PC} 12$ cells against $\mathrm{MPP}^{+}$-induced cell death, the activity of caspase- 3 was measured by ELISA with an ApoAlert caspase-3 assay kit. The PC12 cells exposed 


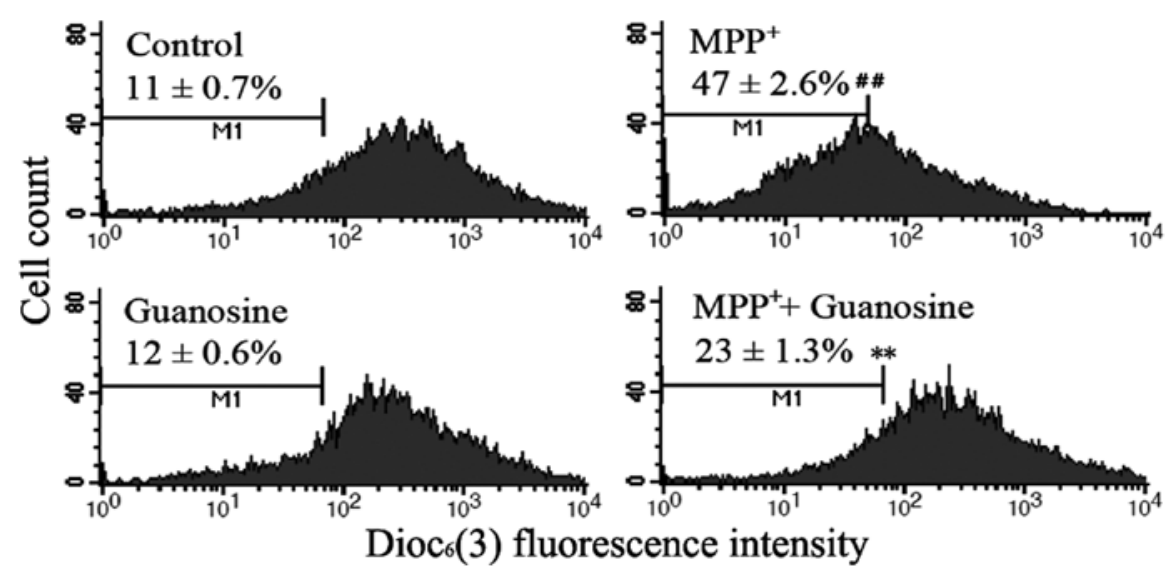

Figure 5. Mitochondrial transmembrane potential was determined by flow cytometry with the mitochondrial dye, 3,3-dihexyloxacarbocyanine iodide [DiOC 6 (3)] The $\mathrm{x}$-axis shows the log scale of fluorescence intensity and the $\mathrm{y}$-axis represents the cell count. Data are presented as the means \pm standard error of the mean (SEM) of 3 independent experiments. ${ }^{\# \#} \mathrm{p}<0.01$ vs. control and ${ }^{* *} \mathrm{p}<0.01$ compared to treatment with 1-methyl-4-phenylpyridinium $\left(\mathrm{MPP}^{+}\right)$.
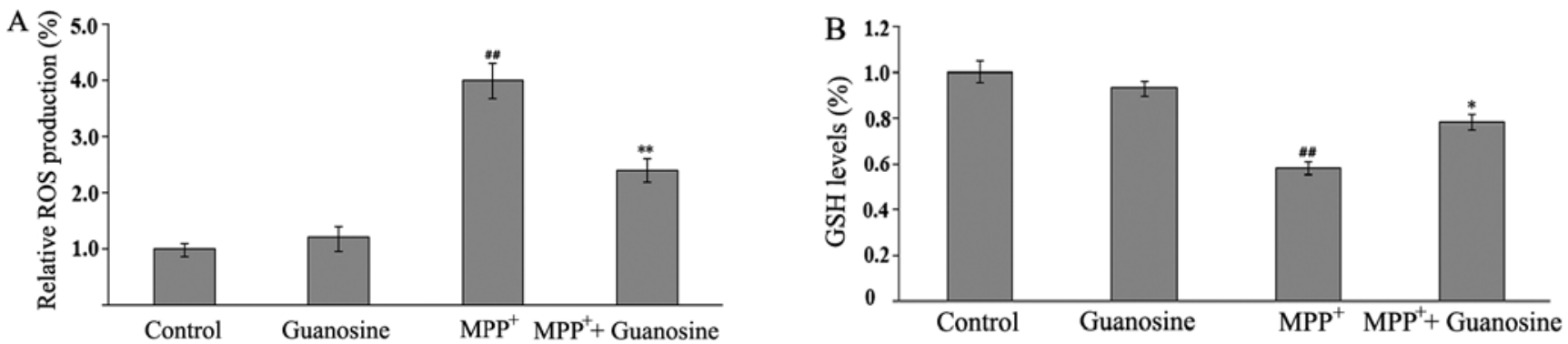

Figure 6. Effects of guanosine on 1-methyl-4-phenylpyridinium (MPP+)-induced reactive oxygen species (ROS) and glutathione (GSH) production. PC12 cells were pre-treated with guanosine for $3 \mathrm{~h}$ and then either exposed or not to $\mathrm{MPP}^{+}$for $72 \mathrm{~h}$. The production of ROS in the cells was measured by flow cytometry with DCFH-DA, and GSH levels were measured by enzyme-linked immunosorbent assay (ELISA). The graphs display the relative levels of (A) ROS production and (B) GSH production compared to the production in the control. Data are presented as the means \pm standard error of the mean (SEM). $\mathrm{n}=3$. \#\# $\mathrm{p}<0.01 \mathrm{vs}$. control and ${ }^{*} \mathrm{p}<0.05$ or ${ }^{* *} \mathrm{p}<0.01$ compared to treatmen wiht $\mathrm{MPP}^{+}$.

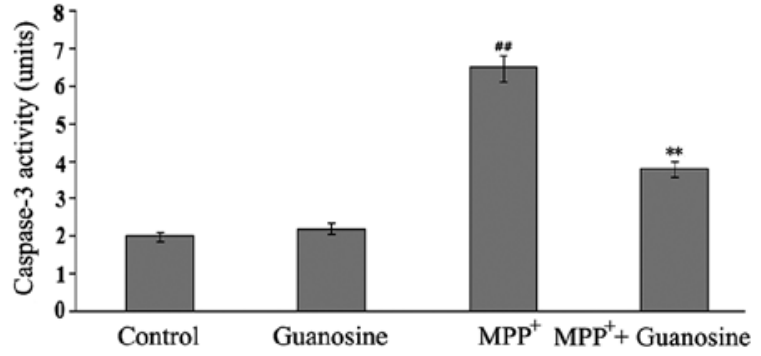

Figure 7. Effects of guanosine on the 1-methyl-4-phenylpyridinium (MPP+)induced caspase- 3 activity in PC12 cells. The cells were either untreated, or treated with $500 \mu \mathrm{M} \mathrm{MPP}^{+}$alone or $500 \mu \mathrm{M} \mathrm{MPP}^{+}$in the presence of $10 \mu \mathrm{M}$ guanosine. Caspase-3 activity was determined by enzyme-linked immunosorbent assay (ELISA). Data are expressed as units for caspase-3 activity, and presented as the means \pm standard error of the mean (SEM). $n=3 .{ }^{\# \#} p<0.01$ vs. control and ${ }^{* *} \mathrm{p}<0.01$ compared to treatment with $\mathrm{MPP}^{+}$.

to $500 \mu \mathrm{M} \mathrm{MPP}+$ showed a significant increase in caspase-3 activity; however, treatment with guanosine alone did not induce any changes in caspase- 3 activity. Pre-treatment with guanosine markedly inhibited the $\mathrm{MPP}^{+}$-induced the increment in caspase-3 activity (Fig. 7), illustrating the protective role of guanosine against $\mathrm{MPP}^{+}$-induced toxicity in PC12 cells.

\section{Discussion}

The non-adenine-based purine, guanosine, is a multifaceted intercellular signaling molecule affecting multiple cellular processes, including cellular growth, differentiation and survival (15). Its protective roles have been reported in previous studies. It protects several cell types against apoptosis induced by a number of agents, such as staurosporine, $\beta$-amyloid and $\mathrm{MPP}^{+}$through its interactions with several steps of the biochemical and cellular cascade $(18,19)$. The protective role of guanosine has also been reported in neurodegenerative diseases (20). The present study demonstrates that guanosine exerts protective effects against apoptotic cell death elicited by $\mathrm{MPP}^{+}$by alleviating mitochondrial dysfunction, inhibiting the activation of caspase- 3 and, subsequently, attenuating cytotoxic cell damage in a reliable cellular model of PD.

$\mathrm{PD}$ is a common neurodegenerative disease clinically characterized by rigidity, resting tremor, bradykinesia and postural instability caused by the degeneration and death of dopaminergic neurons in the pars compacta of the substantia nigra (25). Althought the cellular and molecular events underlying the loss of dopaminergic neurons remain unclear, accumulating evidence indicates that mitochondrial dysfunction may be a central event in the pathogenesis of PD $(2,8)$. The 
mitochondria are the most important cytoplasmic organelles responsible for the life and death of cells (26). The maintenance of membrane potential and the low-conductance of the MPTP are the major properties of mitochondria in living cells, and any changes related to these properties are considered to be critical factors associated with mitochondrial dysfunction and cell death in neurodegenerative diseases $(7,8)$. Multiple proteins are involved in intrinsic apoptotic events associated with mitochondrial dysfunction. The Bcl-2 family of proteins are recognized as key messengers for delivering the apoptotic signal to the mitochondria in response to various insults (27). Pro-apoptotic Bax and anti-apoptotic Bcl-2 are key members of the Bcl-2 family in apoptosis mediated by mitochondrial stress. Under pathogenic conditions, Bax is upregulated and translocates from the cytoplasm to the mitochondria. Once located in the mitochondrial membrane, this protein causes mitochondrial membrane disruption by sequestering Bcl-2 and oligomerizing within the mitochondrial membrane, leading to the opening of the mPTP, the collapse of mitochondrial membrane potential and the subsequent release of pro-apoptotic molecules into the cytoplasm $(28,29)$. Compared to $\mathrm{Bax}, \mathrm{Bcl}-2$ is a key protein that preserves mitochondrial integrity, thereby preventing stressinduced mitochondrial damage in cells (30). Bcl-2 proteins are crucial effectors in the opening of the MPTP and the collapse of mitochondrial potential, thus determining the induction of downstream events in the mitochondrial-dependent cell death pathway, including the release of pro-apoptotic molecules and the activation of caspases (28).

Our results revealed that treatment with $\mathrm{MPP}^{+}$induced the adverse expression levels of two Bcl-2 proteins and the disruption of the mitochondrial membrane potential, supporting the involvement of mitochondrial dysfunction in dopaminergic neuronal degeneration. These changes were reversed by the administration of guanosine prior to exposure to $\mathrm{MPP}^{+}$, demonstrating the protective role of guanosine in mitochondrial-stress induced cell damage, which was partly mediated through the regulation of the expression of proteins involved in the mitochondrial stage of the apoptotic cascade. However, the underlying mechanism responsible for this effect of guanosine is unclear.

A number of studies have indicated that the anti-apoptotic effects of guanosine are mediated by modulating the phosphatidylinositol 3-kinase (PI3K)/Akt/protein kinase B (PKB) and the mitogen-activated protein kinase (MAPK) cell survival pathways $(18,19,31,32)$. PI3K is an upstream signal of glycogen synthase kinase 3 (GSK-3) that plays a central role in the mitochondrial-dependent cell death pathway through the regulation of anti-apoptotic and pro-apoptotic $\mathrm{Bcl}-2$ family proteins, including $\mathrm{Bcl}-2$ and $\mathrm{Bax}$ (33-35). GSK-3 $\beta$ can directly phosphorylate Bax on serine 163 , which results in the activation of Bax, and, subsequently, in its translocation from the cytoplasm to the mitochondria. The inhibition of GSK-3 $\beta$ suppresses the levels of Bax expression, but increases Bcl-2 expression, thereby promoting cell survival by alleviating the mitochondrial dysruption under multiple pathological conditions $(34,36,37)$. Thus, the neuroprotective effects of guanosine may be mediated through the activation of PI3K, which inactivates the downstream signal protein, GSK-3 $\beta$, leading to the attenuation of the opening of the MPTP through the regulation of $\mathrm{Bcl}-2$ family proteins. This hypothesis is surported by our results that guanosine reversed the collapse of mitochondrial membrane potential, the downstream event of the opening of the MPTP in mitochondrial-mediated cell death. The opening of the mPTP causes the collapse of mitochondrial membrane potential and, subsequently, the release of apoptotic proteins from the mitochondria into the cytoplasm. Perhaps the most intriguing pro-apoptotic protein that is released is cytochrome $c$, which triggers the activation of the caspase cascade (28). Caspase-3 is a key effector in the mitochondrial-stress-induced apoptotic pathway, and its activation leads to the irreversible process toward apoptosis $(38,39)$. Our results also demonstrated that guanosine, when administered to $\mathrm{MPP}^{+}$-treated neuronal PC12 cells, effectively prevented the collapse of mitochondrial potential and inhibited caspase- 3 activity, further supporting its protective role in mitichondrial stress-induced neuronal cell damage.

Oxidative stress is another pathological event associated with cell death mechanisms in PD. Studies using postmortem samples of PD have demonstrated that oxidative markers, including soluble protein carbonyl modifications, lipid peroxidation and DNA oxidative damage are selectively observed in the dopaminergic neurons in the pars compacta of the substantia nigra, indicating the correlation of oxidative damage with striatal dopaminergic neurodegeneration $(4,5,40)$. The inhibition of mitochondrial complex I with $\mathrm{MPP}^{+}$and rotenone, wellestablished inducers of Parkinson-like symptoms in humans and primates, can lead to an increase in ROS production and selective dopaminergic neuronal loss in the substantia nigra, supporting the involvement of oxidative stress in the pathogenesis of PD (41). ROS are mainly produced as by-products of oxidative phosphorylation in the mitochondria, and many mitochondrial proteins, which possess iron-sulfur clusters for oxidation-reduction reactions and lack protective histones, are particularly vulnerable to ROS attack (42). Generally, cells develop complex antioxidant systems to scavenge ROS. The GSH protein is recognized as the major non-enzymatic antioxidant in the central nervous system (23). A number of studies have indicated that increased levels of GSH exert protective effects in various neurodegenerative diseases, such as Alzheimer's disease and PD $(22,43,44)$. The reduction of GSH levels contributes to the dysfunction of the mitochondria and increases the sensitivity of neurons to toxic insults (45). The neurotoxin, $\mathrm{MPP}^{+}$, is an inhibitor of the mitochondrial respiratory chain and an inducer of ROS in the mitochondria. Our results revealed that pre-treatment with guanosine reduced the $\mathrm{MPP}^{+}$-induced increase in the production of ROS, the crucial contributors to mitochondrial dysfunction through oxidative damage and the opening of the mPTP. Moreover, guanosine alleviated the decreased levels of GSH induced by the administration of $\mathrm{MPP}^{+}$, reinforcing its protective role in oxidative conditions and its role as a potential neuroprotectant in mitochondrial-mediated neurodegenerative diseases.

In conclusion, this study clearly demonstrates that the neuroprotective effects of guanosine promote dopaminergic neuronal survival by alleviating mitochontrial dysfunction in a cellular model of PD. These neuroprotective effects are partly mediated through the stabilization of mitochondrial membrane potential via the modulation of the expression levels of intrinsic apoptotic proteins involved in the mitochondrial apoptotic pathway. Further studies are required to fully eluci- 
date the mechanisms responsible for the protective effects of guanosine in neurodegenerative diseases, which may promote the development of potentially effective treatments for neurodegenerative diseases by targeting mitochondrias-mediated neuronal damage.

\section{References}

1. Forno LS: Neuropathology of Parkinson's disease. J Neuropathol Exp Neurol 55: 259-272, 1996.

2. Abou-Sleiman PM, Muqit MM and Wood NW: Expanding insights of mitochondrial dysfunction in Parkinson's disease. Nat Rev Neurosci 7: 207-219, 2006.

3. McGeer PL and McGeer EG: Glial reactions in Parkinson's disease. Mov Disord 23: 474-483, 2008.

4. Olanow CW: The pathogenesis of cell death in Parkinson's disease - 2007. Mov Disord 22 (Suppl 17): S335-S342, 2007.

5. Zhou C, Huang Y and Przedborski S: Oxidative stress in Parkinson's disease: a mechanism of pathogenic and therapeutic significance. Ann NY Acad Sci 1147: 93-104, 2008.

6. Li DW, Liu ZQ, Chen W, Yao M and Li GR: Association of glycogen synthase kinase-3 $\beta$ with Parkinson's disease (Review). Mol Med Rep 9: 2043-2050, 2014

7. Vila M and Przedborski S: Targeting programmed cell death in neurodegenerative diseases. Nat Rev Neurosci 4: 365-375, 2003.

8. Perier C, Tieu K, Guégan C, et al: Complex I deficiency primes Bax-dependent neuronal apoptosis through mitochondrial oxidative damage. Proc Natl Acad Sci USA 102: 19126-19131, 2005.

9. Roucou X and Martinou JC: Conformational change of Bax: a question of life or death. Cell Death Differ 8: 875-877, 2001.

10. Shiba-Fukushima K, Imai Y, Yoshida S, et al: PINK1-mediated phosphorylation of the Parkin ubiquitin-like domain primes mitochondrial translocation of Parkin and regulates mitophagy. Sci Rep 2: 1002, 2012.

11. Kopin IJ and Markey SP: MPTP toxicity: implications for research in Parkinson's disease. Annu Rev Neurosci 11: 81-96, 1988.

12. Heikkila RE, Sieber BA, Manzino L and Sonsalla PK Some features of the nigrostriatal dopaminergic neurotoxin 1-methyl-4-phenyl-1,2,3,6-tetrahydropyridine (MPTP) in the mouse. Mol Chem Neuropathol 10: 171-183, 1989.

13. Calon F, Lavertu N, Lemieux AM, et al: Effect of MPTP-induced denervation on basal ganglia $\mathrm{GABA}(\mathrm{B})$ receptors: correlation with dopamine concentrations and dopamine transporter Synapse 40: 225-234, 2001

14. Chiba K, Trevor A and Castagnoli N Jr: Metabolism of the neurotoxic tertiary amine, MPTP, by brain monoamine oxidase. Biochem Biophys Res Commun 120: 574-578, 1984.

15. Rathbone MP, Middlemiss PJ, Gysbers JW, et al: Trophic effects of purines in neurons and glial cells. Prog Neurobiol 59: 663-690, 1999.

16. Dal-Cim T, Martins WC, Santos AR and Tasca CI: Guanosine is neuroprotective against oxygen/glucose deprivation in hippocampal slices via large conductance $\mathrm{Ca}^{2+}$-activated $\mathrm{K}^{+}$channels, phosphatidilinositol-3 kinase/protein kinase $\mathrm{B}$ pathway activation and glutamate uptake. Neuroscience 183: 212-220, 2011.

17. Chang R, Algird A, Bau C, Rathbone MP and Jiang S: Neuro-protective effects of guanosine on stroke models in vitro and in vivo. Neurosci Lett 431: 101-105, 2008.

18. Di Iorio P, Ballerini P, Traversa U, et al: The antiapoptotic effect of guanosine is mediated by the activation of the $\mathrm{PI} 3$-kinase/AKT/PKB pathway in cultured rat astrocytes. Glia 46 356-368, 2004

19. Pettifer KM, Kleywegt S, Bau CJ, et al: Guanosine protects SH-SY5Y cells against beta-amyloid-induced apoptosis. Neuroreport 15: 833-836, 2004.

20. Pettifer KM, Jiang S, Bau C, et al: $\mathrm{MPP}(+)$-induced cytotoxicity in neuroblastoma cells: Antagonism and reversal by guanosine. Purinergic Signal 3: 399-409, 2007.

21. Li DW, Li GR, Lu Y, et al: $\alpha$-lipoic acid protects dopaminergic neurons against $\mathrm{MPP}^{+}$-induced apoptosis by attenuating reactive oxygen species formation. Int J Mol Med 32: 108-114, 2013.

22. Yim SB, Park SE and Lee CS: Protective effect of glycyrrhizin on 1-methyl-4-phenylpyridinium-induced mitochondrial damage and cell death in differentiated PC12 cells. J Pharmacol Exp Ther 321: 816-822, 2007
23. Banerjee R, Vitvitsky V and Garg SK: The undertow of sulfur metabolism on glutamatergic neurotransmission. Trends Biochem Sci 33: 413-419, 2008.

24. Fischer U, Jänicke RU and Schulze-Osthoff K: Many cuts to ruin: a comprehensive update of caspase substrates. Cell Death Differ 10: 76-100, 2003.

25. de Lau LM and Breteler MM: Epidemiology of Parkinson's disease. Lancet Neurol 5: 525-535, 2006.

26. Budd SL and Nicholls DG: Mitochondria in the life and death of neurons. Essays Biochem 33: 43-52, 1998.

27. Akhtar RS, Ness JM and Roth KA: Bcl-2 family regulation of neuronal development and neurodegeneration. Biochim Biophys Acta 1644: 189-203, 2004.

28. Armstrong JS: Mitochondrial membrane permeabilization: the sine qua non for cell death. Bioessays 28: 253-260, 2006.

29. Martinou JC and Green DR: Breaking the mitochondrial barrier. Nat Rev Mol Cell Biol 2: 63-67, 2001.

30. Gollapudi S, McCormick MJ and Gupta S: Changes in mitochondrial membrane potential and mitochondrial mass occur independent of the activation of caspase- 8 and caspase- 3 during CD95-mediated apoptosis in peripheral blood T cells. Int J Oncol 22: 597-600, 2003.

31. Tarozzi A, Merlicco A, Morroni F, et al: Guanosine protects human neuroblastoma cells from oxidative stress and toxicity induced by Amyloid-beta peptide oligomers. J Biol Regul Homeost Agents 24: 297-306, 2010.

32. Molz S, Dal-Cim T, Budni J, et al: Neuroprotective effect of guanosine against glutamate-induced cell death in rat hippocampal slices is mediated by the phosphatidylinositol-3 kinase/Akt/glycogen synthase kinase $3 \beta$ pathway activation and inducible nitric oxide synthase inhibition. J Neurosci Res 89: 1400-1408, 2011.

33. Chuang DM, Chen RW, Chalecka-Franaszek E, et al: Neuro-protective effects of lithium in cultured cells and animal models of diseases. Bipolar Disord 4: 129-136, 2002.

34. Chen RW and Chuang DM: Long term lithium treatment suppresses $\mathrm{p} 53$ and Bax expression but increases Bcl-2 expression. A prominent role in neuroprotection against excitotoxicity. $\mathrm{J}$ Biol Chem 274: 6039-6042, 1999

35. Manji HK and Chen G: PKC, MAP kinases and the bcl-2 family of proteins as long-term targets for mood stabilizers. Mol Psychiatry 7 (Suppl 1): S46-S56, 2002.

36. Chen G, Zeng WZ, Yuan PX, et al: The mood-stabilizing agents lithium and valproate robustly increase the levels of the neuroprotective protein bcl-2 in the CNS. J Neurochem 72: 879-882, 1999.

37. Kaga S, Zhan L, Altaf E and Maulik N: Glycogen synthase kinase-3beta/beta-catenin promotes angiogenic and antiapoptotic signaling through the induction of VEGF, Bcl-2 and survivin expression in rat ischemic preconditioned myocardium. J Mol Cell Cardiol 40: 138-147, 2006.

38. Boatright KM and Salvesen GS: Mechanisms of caspase activation. Curr Opin Cell Biol 15: 725-731, 2003.

39. Kumar S: Caspase function in programmed cell death. Cell Death Differ 14: 32-43, 2007.

40. Navarro A, Boveris A, Bandez MJ, et al: Human brain cortex: mitochondrial oxidative damage and adaptive response in Parkinson disease and in dementia with Lewy bodies. Free Radic Biol Med 46: 1574-1580, 2009.

41. Cassarino DS, Fall CP, Swerdlow RH, et al: Elevated reactive oxygen species and antioxidant enzyme activities in animal and cellular models of Parkinson's disease. Biochim Biophys Acta 1362: 77-86, 1997 .

42. Wallace DC: A mitochondrial paradigm of metabolic and degenerative diseases, aging, and cancer: a dawn for evolutionary medicine. Annu Rev Genet 39: 359-407, 2005.

43. Halliwell B: Oxidative stress and neurodegeneration: where are we now? J Neurochem 97: 1634-1658, 2006.

44. Lee M, Cho T, Jantaratnotai N, Wang YT, McGeer E and McGeer PL: Depletion of GSH in glial cells induces neurotoxicity: relevance to aging and degenerative neurological diseases. FASEB J 24: 2533-2545, 2010.

45. Hall AG: Review: The role of glutathione in the regulation of apoptosis. Eur J Clin Invest 29: 238-245, 1999. 